\title{
A Fast Implicit Algorithm for Highly Magnetized Charged Particle Motion
}

\author{
T.C. Genoni, R.E. Clark and D.R. Welch*
}

Voss Scientific, Albuquerque, NM 87108, USA

\begin{abstract}
We describe a particle advance algorithm for particle-in-cell simulation of highly magnetized charged particles that relaxes the time step constraint due to cyclotron motion. The method preserves the correct cyclotron radius for large time steps and corrects for magnetic field gradients without requiring explicit calculation of the particle magnetic moment. Application of the algorithm is illustrated with electron and ion single particle orbit calculations in a field reversed configuration with rotating magnetic fields. This technique is efficient and applicable to massively parallel simulation.
\end{abstract}

Keywords: Plasma simulation, implicit PIC techniques.

\section{INTRODUCTION}

Charged particle orbits in a magnetic confinement device such as the field-reversed configuration (FRC) are complex and chaotic and require a highly accurate solution technique. Standard explicit particle-in-cell (PIC) techniques making use of the Boris push [1] do not properly describe particle orbits when the product of the cyclotron frequency and time step $\Omega \Delta t$ is significant. While largely accurate in the calculation of $\mathrm{E} \times \mathrm{B}$ drift velocities, explicit PIC over predicts the cyclotron radius and under predicts the gradient $\mathrm{B}$ drift and mirror force with errors scaling roughly as the square of $\Omega \Delta t$. Here, we describe an efficient algorithm that can greatly relax this constraint and is applicable to massively parallel simulation codes.

The magnetic implicit (MI) algorithm described here has similarities to one described by $\mathrm{Vu}$ and Brackbill [2] in that the motion of a charged particle in an electromagnetic field is calculated with the position and relativistic momentum of the particle calculated at the same time level. Recently, Cohen, et al., [3, 4] have reported on a large time step mover which interpolates between a conventional Boris push and particle dynamics in the drift approximation. As with these two algorithms, the MI method preserves the correct particle cyclotron radius for large time steps $(\Omega \Delta t>>1)$. MI differs from these previous schemes in that there is no required explicit calculation of the particle magnetic moment or additional field calculations to determine the local transverse and longitudinal magnetic field gradients. In addition, the usual magnetic field rotation matrix is modified to conserve energy and corrects for the mirror force in regions where a significant longitudinal magnetic field gradient exists. After the particle momentum is advanced, an effective velocity which includes a correction for particle drift in a transverse magnetic field gradient is calculated and used to advance the particle position. To illustrate the application of the algorithm, we present results of electron and ion single particle orbit calculations in an FRC with an odd-parity rotating magnetic field $[5,6]$.

*Address correspondence to this author at the Voss Scientific, Albuquerque, NM 87108, USA; Tel: (505) 255-4201; FAX: (505) 255-4294;

E-mail: dale.welch@vosssci.com

\section{TWO-STEP MI PARTICLE MOVER}

We assume electric and magnetic fields $\mathbf{E}(\mathrm{x}, \mathrm{t})$ and $\mathbf{B}(\mathrm{x}, \mathrm{t})$ as functions of position and time. (In general, these may be functions of the positions and momenta of the charged particles.) At time $t_{n}$ we have the position $\mathbf{r}\left(t_{n}\right)$ and momentum $\mathbf{p}\left(t_{n}\right)\left[\equiv \gamma\left(t_{n}\right) \boldsymbol{\beta}\left(t_{n}\right)\right]$ of the charged particle. For convenience in what follows, we will drop the vector notation and absorb $\mathrm{q} / \mathrm{mc}$ into $\mathrm{E}$ and $\mathrm{B}$ so that they have units of $\mathrm{s}^{-1}$. In the MI algorithm, particle advance is achieved using a two-step predictor-corrector procedure. First we define the vectors

$$
\begin{aligned}
& u=p+\frac{1}{2} E \Delta t, \\
& w=p-\frac{1}{2} E \Delta t,
\end{aligned}
$$

and the standard rotation matrix $\mathrm{M}_{1}$ for the predictor step:

$$
M_{1}=\frac{1}{1+\theta_{1}^{2}}\left(\begin{array}{ccc}
1+\theta_{x}^{2}-\theta_{y}^{2}-\theta_{z}^{2} & 2 \theta_{x} \theta_{y}+2 \theta_{z} & 2 \theta_{x} \theta_{z}-2 \theta_{y} \\
2 \theta_{x} \theta_{y}-2 \theta_{z} & 1+\theta_{y}^{2}-\theta_{x}^{2}-\theta_{z}^{2} & 2 \theta_{y} \theta_{z}+2 \theta_{x} \\
2 \theta_{x} \theta_{z}+2 \theta_{y} & 2 \theta_{y} \theta_{z}-2 \theta_{x} & 1+\theta_{z}^{2}-\theta_{x}^{2}-\theta_{y}^{2}
\end{array}\right)_{t=t_{n}}
$$

where

$\theta_{1}=\frac{|B| \Delta t}{2 \gamma}, \quad \theta_{x, y, z}=\frac{B_{x, y, z} \Delta t}{2 \gamma}$.

The predicted values of particle position and momentum at time $t_{\mathrm{n}+1}$ are obtained according to

$$
\begin{aligned}
& \tilde{w}\left(t_{n+1}\right)=M_{1} u\left(t_{n}\right), \\
& \tilde{p}\left(t_{n+1}\right)=\tilde{w}\left(t_{n+1}\right)+\frac{1}{2} E\left(t_{n}\right) \Delta t, \\
& \tilde{\gamma}\left(t_{n+1}\right)=\left[1+\left|\tilde{p}\left(t_{n+1}\right)\right|^{2}\right]^{1 / 2}, \\
& \tilde{r}\left(t_{n+1}\right)=r\left(t_{n}\right)+\frac{p\left(t_{n}\right)+\tilde{p}\left(t_{n+1}\right)}{\gamma\left(t_{n}\right)+\tilde{\gamma}\left(t_{n+1}\right)} c \Delta t .
\end{aligned}
$$


We use the tilde $(\sim)$ to denote predicted values at $t_{n+1}$ to be used in the corrector step below.

Making use of $\tilde{r}$ we calculate predicted values of $\mathrm{E}$ and $\mathrm{B}$ as

$\tilde{E}=E\left(\tilde{r}, t_{n+1}\right)$,

$\tilde{B}=B\left(\tilde{r}, t_{n+1}\right)$.

For the corrector step, centered values of the fields and particle relativistic factor $\gamma$ are required and these are defined according to

$$
\begin{aligned}
& E\left(t_{n+1 / 2}\right) \equiv \frac{1}{2}\left[\tilde{E}+E\left(t_{n}\right)\right] \\
& B\left(t_{n+1 / 2}\right) \equiv \frac{1}{2}\left[\tilde{B}+B\left(t_{n}\right)\right] \\
& \gamma\left(t_{n+1 / 2}\right) \equiv \frac{1}{2}\left[\tilde{\gamma}+\gamma\left(t_{n}\right)\right] \\
& U \equiv p\left(t_{n}\right)+\frac{1}{2} E\left(t_{n+1 / 2}\right) .
\end{aligned}
$$

Prior to the final particle advance, we transform to a reference frame (denoted by primes) in which $B\left(t_{n+1 / 2}\right)$ is along the $z$-axis. This is carried out using a standard transform matrix $\mathrm{T}$ and indicated schematically below:

$$
\left[\begin{array}{l}
r^{\prime}\left(t_{n}\right) \\
p^{\prime}\left(t_{n}\right) \\
B^{\prime}\left(t_{n}\right) \\
U^{\prime}
\end{array}\right]=T\left[\begin{array}{l}
r\left(t_{n}\right) \\
p\left(t_{n}\right) \\
B\left(t_{n}\right) \\
U
\end{array}\right]
$$

We will also need

$$
\left[\begin{array}{c}
\tilde{p}^{\prime}\left(t_{n+1}\right) \\
\tilde{B}^{\prime}\left(t_{n+1}\right)
\end{array}\right]=T\left[\begin{array}{c}
\tilde{p}\left(t_{n+1}\right) \\
\tilde{B}\left(t_{n+1}\right)
\end{array}\right] \text {. }
$$

Now we define the rotation matrix $\mathrm{M}_{2}$ in terms of $B\left(t_{n+1 / 2}\right)$,

$$
M_{2}=\frac{1}{1+\theta_{2}^{2}}\left(\begin{array}{ccc}
\alpha\left(1-\theta_{2}^{2}\right) & 2 \alpha \theta_{2} & 0 \\
-2 \alpha \theta_{2} & \alpha\left(1-\theta_{2}^{2}\right) & 0 \\
0 & 0 & \Gamma\left(1+\theta_{2}^{2}\right)
\end{array}\right)
$$

where

$\theta_{2}=\frac{\left|B\left(t_{n+1 / 2}\right)\right| \Delta t}{2 \gamma\left(t_{n+1 / 2}\right)}$.

In Eq. (10),

$$
\Gamma=1+\frac{\Omega_{e f f} \Delta t}{U_{z}^{\prime}}
$$

and

$$
\Omega_{e f f}=\frac{1}{2 \gamma\left(t_{n+1 / 2}\right)}\left\{\left[p_{x}^{\prime} B_{y}^{\prime}-p_{y}^{\prime} B_{x}^{\prime}\right]_{t=t_{n}}+\left[\tilde{p}_{x}^{\prime} \tilde{B}_{y}^{\prime}-\tilde{p}_{y}^{\prime} \tilde{B}_{x}^{\prime}\right]_{t=t_{n+1}}\right\} .
$$

The factor $\Gamma$ in Eq. (10) accounts for the mirror force on the particle due to the longitudinal field gradient and is discussed below and illustrated in Sec. III.c for electron motion in an FRC.

The corrector step advance of the particle momentum proceeds as follows:

$w^{\prime}\left(t_{n+1}\right)=M_{2} U^{\prime}$,

$p^{\prime}\left(t_{n+1}\right)=w^{\prime}\left(t_{n+1}\right)+U^{\prime}-p^{\prime}\left(t_{n}\right)$,

$\gamma\left(t_{n+1}\right)=\left[1+\left|p^{\prime}\left(t_{n+1}\right)\right|^{2}\right]^{1 / 2}$.

The factor $\alpha$ in matrix $M_{2}$ is specified according to

$\alpha^{2}=\frac{U_{x}^{\prime 2}+U_{y}^{\prime 2}+U_{z}^{\prime 2}-\left(\Gamma U_{z}^{\prime}\right)^{2}}{U_{x}^{\prime 2}+U_{y}^{\prime 2}}$,

which insures that $\mid w^{\prime}\left(t_{n+1}|=| U^{\prime} \mid\right.$ so that

$\gamma\left(t_{n+1}\right)-\gamma\left(t_{n}\right)=\frac{p^{\prime}\left(t_{n+1}\right)+p^{\prime}\left(t_{n}\right)}{\gamma\left(t_{n+1}\right)+\gamma\left(t_{n}\right)} E^{\prime}\left(t_{n+1 / 2}\right) \Delta t$,

and energy is conserved in the corrector step push.

We now calculate a drift velocity correction due to the transverse gradient of $\mathrm{B}$ in the primed coordinate frame. It can be shown that on average over a large number of time steps, the following expressions give the correct drift velocity corrections for moderate (this will be quantified below) transverse magnetic field gradients:

$\beta_{d x}^{\prime}=\frac{-\left[\frac{\tilde{p}_{x}^{\prime}\left(t_{n+1}\right)}{\tilde{\gamma}\left(t_{n+1}\right)}-\frac{p_{x}^{\prime}\left(t_{n}\right)}{\gamma\left(t_{n}\right)}\right]\left[\tilde{B}_{z}^{\prime}\left(t_{n+1}\right)-B_{z}^{\prime}\left(t_{n}\right)\right]}{4\left|B\left(t_{n+1 / 2}\right)\right|}$,

$\beta_{d y}^{\prime}=\frac{-\left[\frac{\tilde{p}_{y}^{\prime}\left(t_{n+1}\right)}{\tilde{\gamma}\left(t_{n+1}\right)}-\frac{p_{y}^{\prime}\left(t_{n}\right)}{\gamma\left(t_{n}\right)}\right]\left[\tilde{B}_{z}^{\prime}\left(t_{n+1}\right)-B_{z}^{\prime}\left(t_{n}\right)\right]}{4\left|B\left(t_{n+1 / 2}\right)\right|}$.

We define an effective velocity

$\beta_{e f f}^{\prime}=\beta_{d}^{\prime}+\frac{p^{\prime}\left(t_{n}\right)+p^{\prime}\left(t_{n+1}\right)}{\gamma\left(t_{n}\right)+\gamma\left(t_{n+1}\right)}$

and advance the particle position according to

$r^{\prime}\left(t_{n+1}\right)=r^{\prime}\left(t_{n}\right)+\beta_{e f f}^{\prime} c \Delta t$

The final step is transformation back to the lab frame using the matrix $\mathrm{T}^{-1}$.

The parallel dynamics portion of the MI algorithm was tested extensively with single particle orbit calculations in a model magnetic field of the form

$B_{x}=-\frac{B_{0}}{2} \frac{x}{L_{\|}}$,

$B_{y}=-\frac{B_{0}}{2} \frac{y}{L_{\|}}$,

$B_{z}=B_{0}\left[1+\frac{z-z_{0}}{L_{\|}}\right]$ 
where $B_{0}$ is the characteristic magnetic field strength and scale length $L_{\|}\left(=\left|\nabla_{\|} B / B\right|\right)$ is a measure of the strength of the longitudinal field gradient. The particle was injected into the mirror field configuration with initial velocity components $v_{\perp}{ }^{0}$ and $v_{\mathrm{z}}{ }^{0}$ and its motion followed until its longitudinal velocity reversed and the particle returned to its initial position. The velocity and magnetic field parameters were all varied over an order of magnitude, and the orbits calculated with the MI algorithm compared to "exact" calculations with a fourth-order Runga-Kutta (RK4) differential equation solver with adaptive step size control and a relative error tolerance of $10^{-9}$. It was found that the MI solver reproduced the correct particle motion for large time steps $(\Omega \Delta t>>1)$ with the restriction that the product of $v_{\mathrm{z}}{ }^{0} \Delta t<0.05 L_{\|}$.

For particle motion in a transverse magnetic field gradient, it was found that the maximum allowable time step is limited by the ratio $r_{L} / L_{\perp}$ where $r_{L}$ is the cyclotron radius and $L_{\perp}(=|\nabla \perp B / B|)$ is a measure of the strength of the transverse gradient. As one would expect, the larger the ratio, the smaller the time step required to accurately reproduce the correct transverse particle drift. Table $\mathbf{1}$ summarizes the results from a large number of MI calculations for magnetic fields of the simple form $B_{z}=B_{0}$ $\left(1+x / L_{\perp}\right)$.

Table 1. Approximate Values of Maximum Allowable Time Step for Various Values of $r_{L} / L_{\perp}$

\begin{tabular}{|c|c|}
\hline $\mathbf{r}_{\mathbf{L}} / \mathbf{L}_{\perp}$ & $(\boldsymbol{\Omega \Delta} \mathbf{t})_{\max }$ \\
\hline \hline 0.10 & 3 \\
\hline 0.05 & 6 \\
\hline 0.02 & 15 \\
\hline 0.01 & 30 \\
\hline
\end{tabular}

An illustration of the calculation of transverse electron drift in a reversed field configuration magnetic field is given in Sec. III.d.

\section{PARTICLE ORBITS IN A FRC WITH ROTATING MAGNETIC FIELD}

References [5] and [6] present a detailed study of ion and electron heating in the FRC with an odd polarity rotating magnetic field $\left(\mathrm{RMF}_{\mathrm{o}}\right)$ which is being considered for fusion reactor application. In this section, we present a few results for electron and ion orbits in such a field configuration calculated using the MI particle mover algorithm described in the previous section. In each case, MI results will be compared to results obtained with the RK4 solver. Specifics of the FRC confining field and the $\mathrm{RMF}_{\mathrm{o}}$ are discussed below. Results presented in Secs. III.c and III.d are for the magnetostatic FRC only, and illustrate the calculation of electron motion in regions of longitudinal and transverse magnetic field gradients. Electron and ion heating in an $\mathrm{RMF}_{\mathrm{o}}$ are also considered in Secs. III.e and III.f, respectively.

\section{a. Confining FRC Field}

Following Refs. [5, 6], we use the Solov'ev model based on the assumption of a scalar pressure within the plasma.
The azimuthally symmetric equilibrium fields within the plasma can be derived from the vector potential $A_{\varphi}$ given by

$r A_{\phi}=B_{a} \frac{r^{2}}{2}\left(1-\frac{r^{2}}{r_{s}^{2}}-\frac{z^{2}}{z_{s}^{2}}\right)$,

where $r$ and $z$ refer to a standard $(r, \varphi, z)$ cylindrical coordinate system (see Fig. 1), and $r_{s}$ and $z_{s}$ determine the radial and longitudinal extent of the rotating ellipsoidal plasma. $B_{a}$ is the maximum field at $r=z=0$. The vector potential in Eq. (20) derives from a rigid-rotor (linear in $r$ ) plasma current density. External to the plasma, we use the solution for the fields presented in [7].

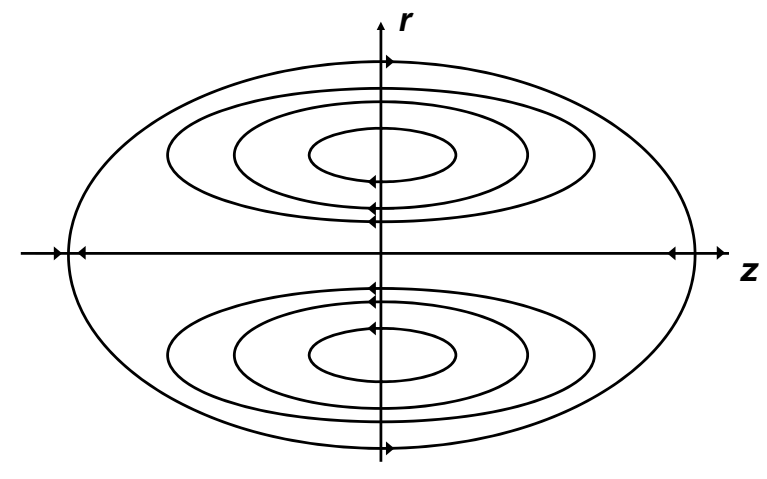

Fig. (1). The FRC coordinate system used in the orbit calculations.

\section{b. Odd-Parity Rotating Magnetic Field}

The $\mathrm{RMF}_{\mathrm{o}}$ fields used here are derived from the vector potential given by

$$
\begin{aligned}
& A_{r}=\frac{2 B_{o}}{k} I_{0}(k r) \cos (k z) \cos \Psi, \\
& A_{\phi}=\frac{-2 B_{o}}{k} I_{0}(k r) \cos (k z) \sin \Psi, \\
& A_{z}=\frac{-2 B_{o}}{k} I_{1}(k r) \sin (k z) \cos \Psi,
\end{aligned}
$$

where $\Psi=\phi-\omega t, B_{o}$ is the amplitude of the $\mathrm{RMF}_{\mathrm{o}}$ with angular frequency $\omega$, and $k$ determines the wavelength in $z$. (The vector potential of Eq. (21) may be obtained by setting the phase $\Psi_{0}=\pi / 2$ in Eq. 8 of Ref. [5].)

In the calculations below, we follow an example from [5] and use $r_{s}=10 \mathrm{~cm}, z_{s}=50 \mathrm{~cm}$ (elongation $\kappa=z_{s} / r_{s}=5$ ), and a peak FRC field of $B_{a}=2 \times 10^{4} \mathrm{G}$. This corresponds to peak electron and ion cyclotron frequencies of

$$
\Omega_{e a}=\frac{e B_{a}}{m_{e} c}=3.52 \times 10^{11} s^{-1}
$$

and

$\Omega_{i a}=\frac{e B_{a}}{m_{i} c}=9.78 \times 10^{7} \mathrm{~s}^{-1}$,

where $m_{i}=2 m_{p}$ for deuterium ions.

For the $\mathrm{RMF}_{\mathrm{o}}$ fields we take $\omega=0.8 \Omega_{\mathrm{ia}}, k=\pi / 2 z_{s}$, and $B_{o}=20 \mathrm{G}$ or 0.1 percent of $B_{a}$. In all cases the particle energy is initialized to $100 \mathrm{eV}$, except in the electron 
transverse gradient drift case where $1-\mathrm{keV}$ electrons are assumed. The results presented below are obviously not intended to be an exhaustive study of electron and ion heating in this field configuration, but instead were chosen as a limited set of illustrations of the application of the MI algorithm.

\section{c. Mirror Force on Electron}

For this calculation, we consider the magnetostatic FRC confining field only, and initialize the $100-\mathrm{eV}$ electron in a region of increasing $B_{z}$ at $r_{0}=7.07$ and $z_{0}=-30$, with initial velocity in the $+z$ direction. Fig. (2) shows the $z$ motion of the electron as a function of $\tau\left(=\Omega_{\mathrm{ea}} t\right)$. A time step of $\Omega_{\mathrm{ea}} \Delta t=$ 100 was used for the MI calculation, which corresponds to a local $\Omega_{\mathrm{e}} \Delta t$ of about 63 . The electron moves in the $+z$ direction until turned around by the mirror $B_{z}$ field. The second curve of Fig. (2) calculated using MI is nearly indistinguishable from the "exact" curve obtained from RK4. For comparison to a standard PIC push with large time step, the MI calculation without the mirror field correction $[\Gamma=\alpha$ $=1$ in Eq. (10)] is shown.

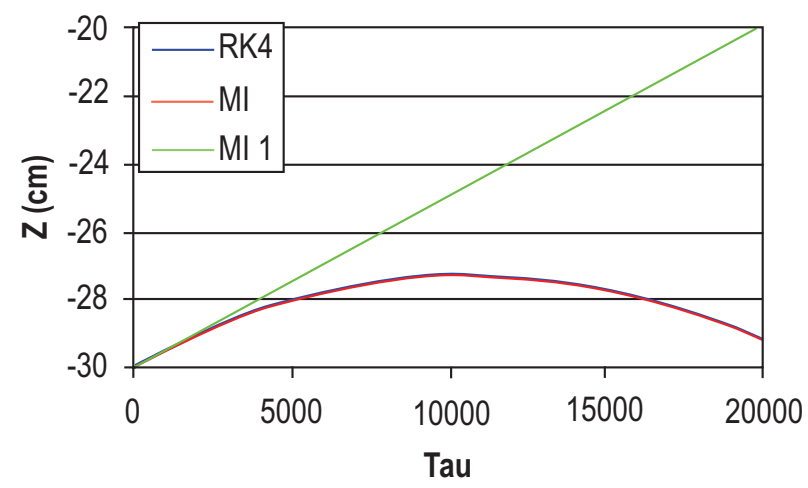

Fig. (2). Electron motion in converging field as calculated with RK4 in blue, MI in red, and MI with $\Gamma=\alpha=1$ (no mirror force correction) in green.

\section{d. Electron Transverse Gradient Drift}

We again consider the magnetostatic FRC only and initialize a $1-\mathrm{keV}$ electron in a region of transverse magnetic field gradient in the $\mathrm{x}$ direction at $x_{0}=6, y_{0}=z_{0}=0$, and with initial velocity in the $+\mathrm{x}$ direction. The ratio $r_{L} / L_{\perp}$ for these parameters is approximately 0.016 . We anticipate cyclotron motion in the $x, y$ plane with transverse drift in the $y$ direction. For the MI calculation we set $\Omega_{\mathrm{ea}} \Delta t=50$, which corresponds to a local $\Omega_{\mathrm{e}} \Delta t \sim 14$. The calculated electron drifts are shown in Fig. (3b) from RK4 and from MI with and without the $\beta_{d}$ correction to the effective velocity of Eq. (18). The time step required to accurately reproduce the drift agrees approximately with the results of Table 1 . The corresponding $\mathrm{x}$-motion as a function of $\tau$ is shown in Fig. (3a).

\section{e. Electron Heating in $\mathbf{R M F}_{\mathbf{o}}$}

In Ref. [5], it was shown that significant electron heating occurs for electrons initialized in the vicinity of the magnetic field null at $r_{0}=7.07, z_{0}=0$. We first consider the orbit of a single $100-\mathrm{eV}$ electron initialized at $x_{0}=7.07, y_{0}=z_{0}=0$, with initial velocity $\mathrm{v}_{0}$ corresponding to $100 \mathrm{eV}$ and $v_{x}=$ $v_{0} / 2, v_{y}=v_{0} / 2$ and $v_{z}=v_{0} / 2^{1 / 2}$. The time step for the MI calculation here was $\Omega_{\mathrm{ea}} \Delta t=4$ and was found to be adequate to resolve the electron motion in the combined FRC and $\mathrm{RMF}_{\mathrm{o}}$ fields. Fig. (4a) shows the projection of the electron orbit in the $x y$ plane for $\Omega_{e a} t=0$ to $\Omega_{e a} t=2 \pi \times 10^{3}$. In Fig. (4b) the same projection is shown for the time interval $(0,2 \pi$ $x 10^{4}$ ). Here we see that the orbits differ from one another due in large part to the chaotic nature of the orbits at late times which was illustrated and discussed in detail in Ref. [5].

We next calculate the maximum energy transfer given to $100-\mathrm{eV}$ electrons initialized near the FRC null at $r_{0}=7.07, z_{0}$ $=0$. For these calculations we set $y_{0}=z_{0}=0$ and initial positions $x_{0}=f(7.07)$ with values of $f$ between 0.88 and 1.12. For each $x_{0}$ position, we consider 128 sets of initial velocities given by

$$
\begin{aligned}
& v_{x}=v_{0} \sin \theta_{0} \cos \phi_{0} \\
& v_{y}=v_{0} \sin \theta_{0} \sin \phi_{0} \\
& v_{z}=v_{0} \cos \theta_{0},
\end{aligned}
$$

with 8 values of $\theta_{0}$ on the interval $(0, \pi)$ and 16 values of $\phi_{0}$ on $(0,2 \pi)$. For each orbit, the maximum energy obtained by the electron over the $\tau\left(=\Omega_{e a} t\right)$ interval $(0,2000 \times 2 \pi)$ was calculated, and at each $\mathrm{x}_{0}$ the energy was averaged over the 128 sets of initial velocities. Results are shown in Fig. (5) as
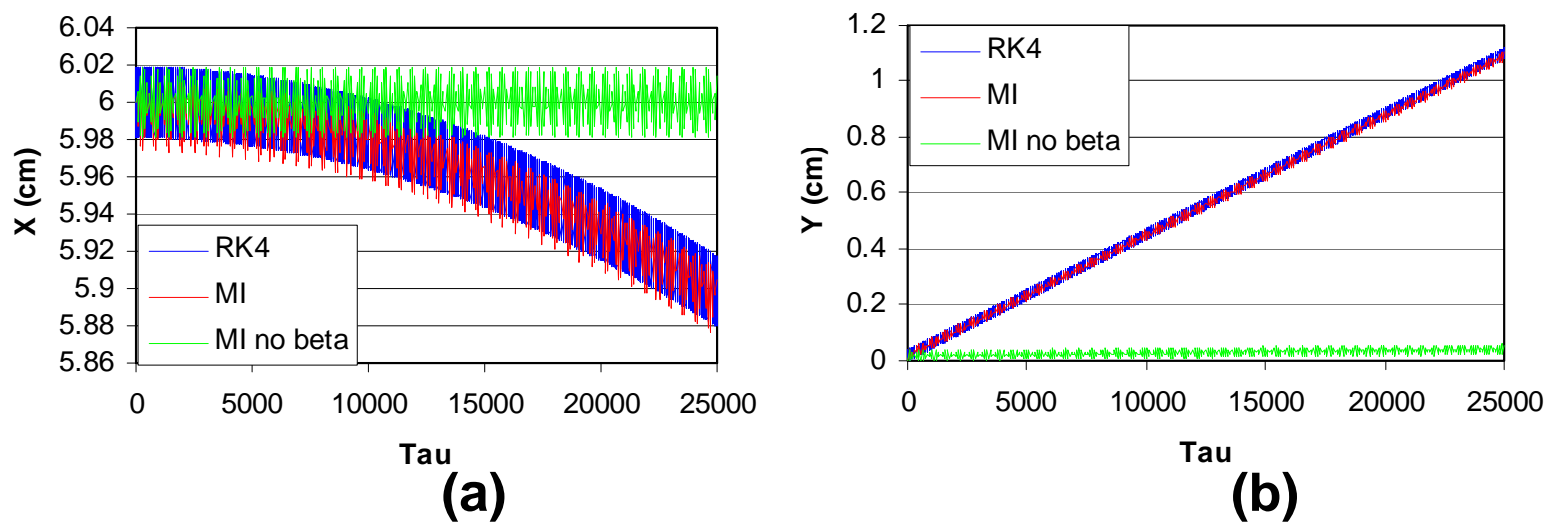

Fig. (3). The $x$ (a) and $y$ (b) motion (drift) of electron in transverse $B_{z}$ gradient as calculated by RK4, MI, and MI with $\beta_{d}=0$ (label MI no beta). 


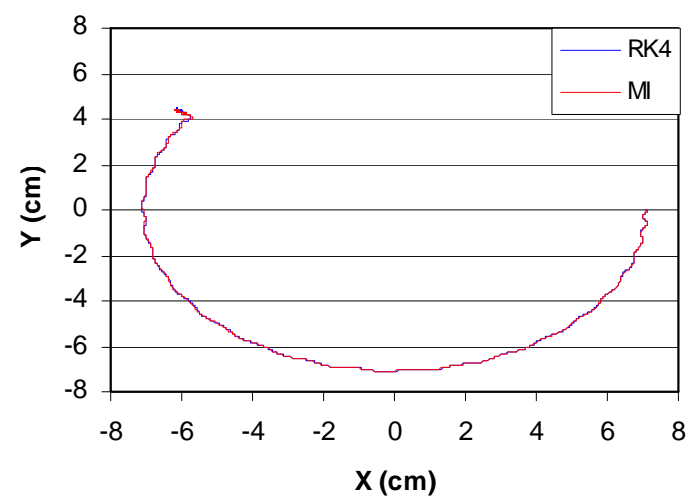

(a)

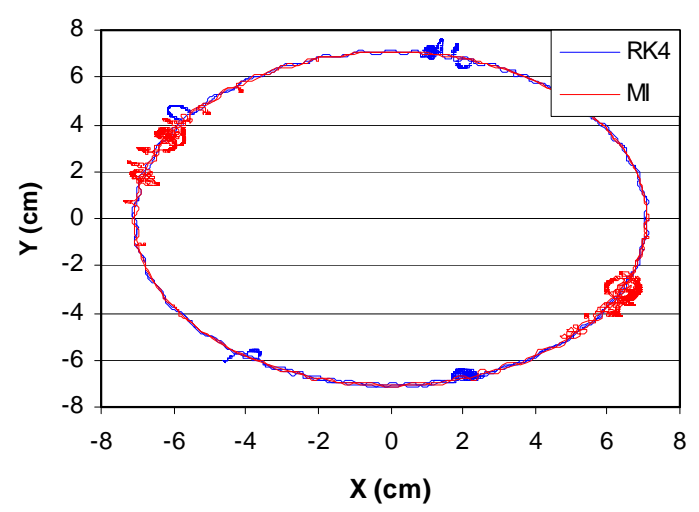

(b)

Fig. (4). Electron orbit in combined FRC and $\mathrm{RMF}_{\mathrm{o}}$ fields for (a) $\Omega_{\mathrm{eo}} t=\left(0,2 \pi \times 10^{3}\right)$ and (b) $\Omega_{\mathrm{eo}} t=\left(0,2 \pi \times 10^{4}\right)$ as calculated by RK4 and MI with $\Omega_{\mathrm{eo}} \Delta t=4$.

a function of the parameter $f$ and excellent agreement between the RK4 and MI calculations is apparent. The same qualitative behavior is observed in figure 11 of [5]. $\Omega_{e a} \Delta t=4$ corresponds to a time step of $\Delta t=0.0114 \mathrm{~ns}$ and is the limiting value (maximum allowable to resolve the $\mathrm{RMF}_{\mathrm{o}}$ frequency) for the MI calculations of electron heating.

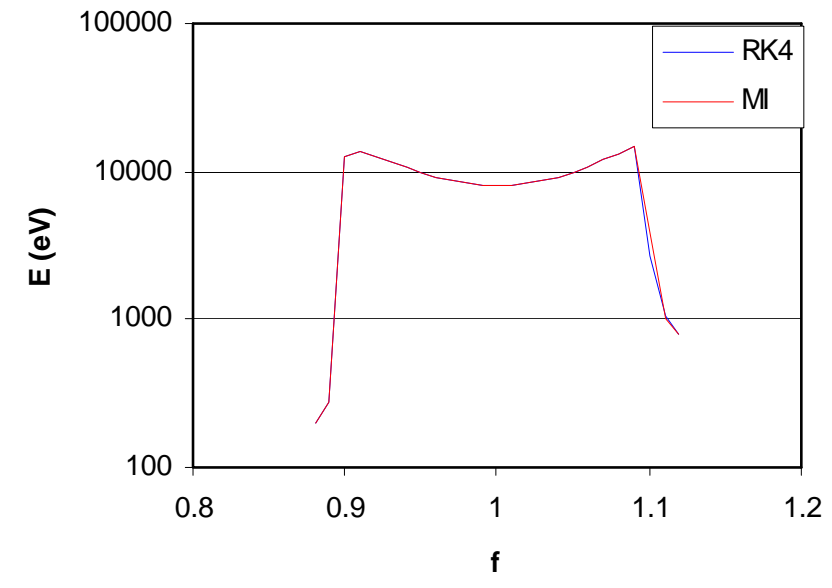

Fig. (5). With the $\mathrm{RMF}_{\mathrm{o}}$ fields, the maximum energy (average) given to electrons initialized near the magnetic field null in the FRC, as calculated by RK4 and MI.

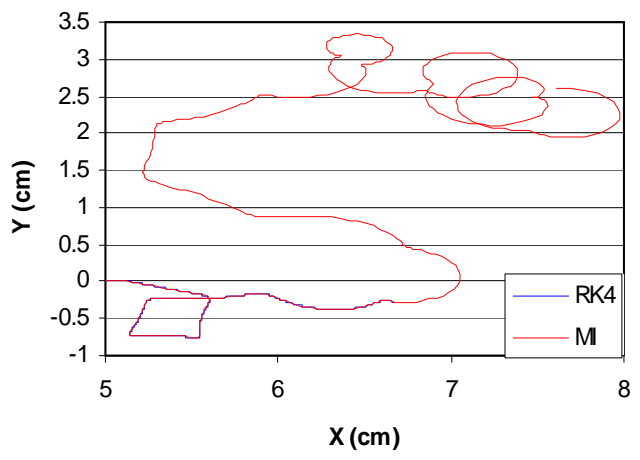

(a)

\section{f. Ion Heating in $\mathbf{R M F}_{\mathbf{0}}$}

For the deuterium ions $\left(\mathrm{m}_{\mathrm{i}}=2 \mathrm{~m}_{\mathrm{p}}\right)$ we considered a typical orbit for a 100-eV ion initialized at $x_{0}=5, z_{0}=20$ with initial velocity $v_{x}=v_{0} / 2, v_{y}=v_{0} / 2$, and $v_{z}=v_{0} / 2^{1 / 2}$. The $x y$ plane projection of the orbit for the time interval $\Omega_{\mathrm{ia}} t=(0$, $25 \times 2 \pi$ ) is shown in Fig. (6a) for the RK4 and MI calculations. A time step of $\Omega_{\mathrm{ia}} \Delta t=1 / 3$ was required to adequately resolve the time-dependence of the $\mathrm{RMF}_{\mathrm{o}}$. Fig. (6b) shows the orbit projection for the time interval $(0,1000$ $\times 2 \pi)$. The RK4 and MI orbits are qualitatively similar but again differ in detail due to chaotic late time behavior.

We next calculated maximum energy transfer given to an ion initialized at $x_{0}=5, y_{0}=0, z_{0}=20$, and averaged over 128 sets of initial velocities with $v_{0}$ in Eq. (23) corresponding to the $100-\mathrm{eV}$ deuterium ion. The calculated values of maximum (average) energy transfer for the time interval $\Omega_{\mathrm{ia}} \mathrm{t}$ $=(0,2000 \times 2 \pi)$ were $6.55 \times 10^{4} \mathrm{eV}$ and $6.66 \times 10^{4} \mathrm{eV}$ with MI and RK4, respectively, in excellent agreement and consistent with figure 8 of Ref. [5].

During the course of performing ion orbit calculations, it was observed that ions initialized near the $z$-axis had a significant probability of escaping the plasma region (defined here as reaching an $r$ position $\geq 1.5 r_{s}$ or a $\mathrm{z}$ position $\geq 1.5 z_{s}$ ). To illustrate this, we performed orbit calculations for $100-\mathrm{eV}$ ions initialized at $x_{0}=1, y_{0}=0$, and eight values of $z_{0}$ on the interval $(-40,40)$. At each z position, 32 sets of

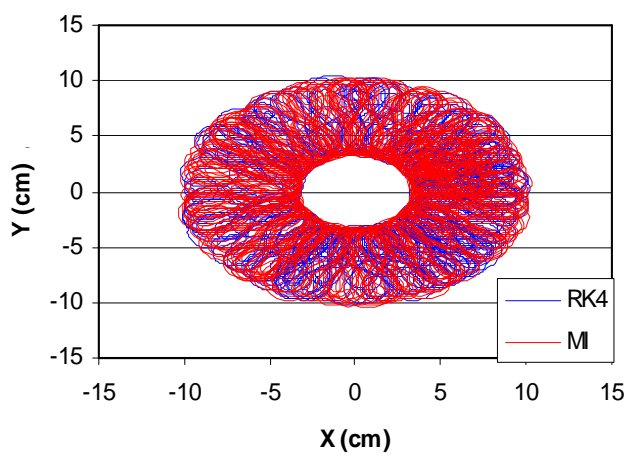

(b)

Fig. (6). Ion orbit in combined FRC and $\mathrm{RMF}_{\mathrm{o}}$ fields for the time interval (a) $\Omega_{i a} t=(0,25 \times 2 \pi)$ and (b) $\Omega_{i a} t=(0,1000 \times 2 \pi)$ as calculated by RK4 and MI with $\Omega_{i a} \Delta t=1 / 3$. 
initial conditions were chosen corresponding to Eq. (23) with four values of $\theta_{0}$ on $(0, \pi)$ and eight values of $\phi_{0}$ on $(0,2 \pi)$. For the interval $\Omega_{\mathrm{ia}} \Delta t=(0,200 \times 2 \pi)$, it was observed that about $1 / 2$ of the 256 orbits resulted in an escape as defined above (127 using RK4 and 130 with MI).

\section{SUMMARY AND CONCLUSIONS}

We have developed an efficient algorithm for highly magnetized charged particle motion that, within reasonable restrictions, allows the use of large time steps $(\Omega \Delta t>>1)$ while preserving (on average) the correct particle gyroradius and energy. The method accurately accounts for transverse and longitudinal magnetic field gradients without requiring explicit calculation of the particle magnetic moment or additional field calculations to determine the local gradients. The examples given here include electron and ion motions in complex magnetic field configurations and dynamic electromagnetic fields but ignore self field contributions (single particle orbits only). Recent preliminary tests of plasmas drifting into high field solenoids including self fields have shown encouraging results indicating the algorithm is stable given resolution of the plasma frequency. Future work will include implementation of the scheme in a format that is fully implicit for the particle motion and relaxes both the cyclotron and plasma frequency time step constraints.

\section{REFERENCES}

[1] Boris, JP. Relativistic plasma simulation - optimization of a hybrid code. Boris JP, Shanny R, Eds. In: $4^{\text {th }}$ conference on numerical simulation of plasmas. Washington DC: Naval Research Laboratory 1970; pp. 3-67.

[2] $\mathrm{Vu} \mathrm{HX}$, Brackbill JU. Accurate numerical solution of charged particle motion in a magnetic field. J Comp Phys 1995; 116: 384-7.

[3] Cohen RH, Friedman A, Kireef Covo KM, et al. Simulating electron clouds in heavy-ion accelerators. Phys Plasmas 2005; 12: 056708 .

[4] Cohen RH, Friedman A, Grote DP, Vay J-L. Large-timestep mover for particle simulations of arbitrarily magnetized species. Nucl Instrum Methods Phys Res A 2007; 577: 52-7.

[5] Glasser AH, Cohen SA. Ion and electron acceleration in the fieldreversed configuration with an odd-parity rotating magnetic field. Phys Plasmas 2002; 9: 2093-2102.

[6] Cohen SA, Glasser AH. Ion heating in the field-reversed configuration by rotating magnetic fields near the ion-cyclotron resonance. Phys Rev Lett 2000; 85: 5114-7.

[7] Zakharov L, Shafranov V. Equilibrium of current-carrying plasmas in toroidal configurations. In: Leontovich MA, Ed. Reviews of Plasma Physics. New York: Consultants Bureau 1986; vol. 11: pp. 206-11. 